\section{Natali Chávez Vereau ${ }^{1}$ Marco Alarcón Palacios ${ }^{2}$}

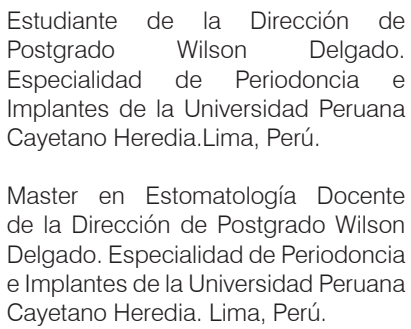

Master en Estomatología Docente de la Dirección de Postgrado Wilson Delgado. Especialidad de Periodoncia e Implantes de la Universidad Peruana Cayetano Heredia. Lima, Perú.

\section{Correspondencia:}

Natali Chávez Vereau

Jr. Angamos Este 245 Miraflores. Lima Perú.

Teléfono: 511-2413910

E-mail: natali.chavez.v@upch.pe oecasa@hotmail.com

\title{
Enfermedad gingival en adolescentes: Diagnóstico y tratamiento.
}

Chavez-Vereau $N^{1}$, Alarcón-Palacios $M^{2}$. Enfermedad gingival en adolescentes, diagnóstico y tratamiento. Rev Estomatol Herediana. 2012; 22(3): 167-70.

\section{RESUMEN}

La pubertad es la primera fase de la adolescencia, en la cual se producen las modificaciones propias del paso de la infancia a la edad adulta. Dentro de la enfermedad gingival inducida por placa se encuentra la enfermedad gingival modificada por factores sistémicos; la que involucra a su vez a la gingivitis asociada con la pubertad. Una serie de parámetros clínicos se identifican al examen inicial como son: encía marginal y papilar edematosa y eritematosa, sangrado al sondeo y deficiencia en la higiene oral por la molestia que causa el cepillado. El objetivo del presente reporte es demostrar que con la fase inicial de tratamiento se puede dar mejoría a los tejidos gingivales, sumado con una buena instrucción de higiene oral del paciente con la utilización de elementos complementarios que influyan en el control bacteriano de la zona; de esta manera confirmar que un adecuado mantenimiento y control de los tejidos hará que los pacientes en fase puberal puedan mantener buena salud en los tejidos periodontales.

\section{Palabras claves: GINGIVITIS, HIGIENE ORAL, FACTORES DE ENFERMEDAD GINGIVAL.}

Gingival diseases in adolescents: Diagnosis and treatment.

\section{ABSTRACT}

Puberty is the first phase of adolescence, in which changes occur of the transition from childhood to adulthood. The section on dental Plaque-Induced Gingival Diseases includes gingival diseases modified by systemic factors, which in turn involves puberty-associated gingivitis. A number of clinical parameters were identified at initial examination are marginal and papillary gingiva edematous and erythematous, bleeding on probing, and poor oral hygiene for the inconvenience caused in the brush. The goal of this report is to show that the initial phase of treatment can be given to improving the gingival tissues, coupled with good patient instruction regarding oral hygiene with the use of complementary elements that influence the bacterial control of the area, thus confirming that proper maintenance and control of the tissues will pubertal stage patients can maintain good health in periodontal tissues.

Key words: GINGIVITIS, ORAL HYGIENE, GINGIVAL DISEASE FACTORS.

\section{Introducción.}

Las enfermedades gingivales presentan varias características clínicas comunes, dentro de las cuales encontramos signos clínicos de inflamación los cuales se limitan a la encía, presencia de placa bacteriana iniciando y/o exacerbando la severidad de la enfermedad, pero teniendo en cuenta que esta enfermedad es reversible mediante la eliminación del factor etiológico. ${ }^{1}$

La definición y clasificación de las enfermedades gingivales no ha sido una tarea fácil. Las herramientas y los métodos para identificarlas han variado en función a los criterios utilizados por los epidemiólogos, investigadores, o la práctica clínica. Esta clasificación se basó en estudios experimentales y/o epidemiológicos humanos que evaluaron con precisión y fiabilidad una base funcional de trastorno que fue localizado en la encía. ${ }^{1,2}$

La clasificación de las enfermedades gingivales que depende de la placa dental para iniciar la enfermedad ha sido clasificada en dos grupos. Las dos categorías de enfermedad gingival inducida por placa son las afectadas por factores locales y las que se ven afectadas tanto por factores locales y modificados por factores sistémicos específicos del huésped. En este reporte de caso se presenta las características clínicas de la enfermedad gingival induci- da por placa así como la influencia de factores hormonales correspondientes a la edad. ${ }^{2,3}$

\section{Gingivitis asociado a la pubertad}

La pubertad es el complejo proceso de maduración sexual dando lugar a un individuo capaz de reproducirse, no es un episodio aislado, sino más bien es una fase endocrinológica de eventos que producen cambios en el aspecto físico y en el comportamiento de los adolescentes. ${ }^{4}$

La incidencia y la gravedad de la gingivitis en los adolescentes se ven influidos por una variedad de factores, incluyendo los niveles de placa, caries dental, respiración por 
la boca, el apiñamiento y malposición dental. Sin embargo, el aumento de los niveles de esteroides hormonales durante la pubertad en ambos sexos tiene un efecto transitorio sobre el estado inflamatorio de la encía. ${ }^{1,5}$

Un número de estudios ha demostrado un aumento de la inflamación gingival en personas en etapa circumpuberal en ambos sexos, sin un concomitante aumento de los niveles de placa. La enfermedad gingival asociada a la pubertad tiene muchas de las características clínicas de la gingivitis inducida por placa, que se caracteriza por la propensión a desarrollar signos de inflamación gingival en la presencia de cantidades relativamente pequeñas de placa durante el período circumpuberal. (Tabla 1) ) $^{1,6}$

\section{Reporte de caso}

Paciente de 15 años de edad, de sexo femenino en aparente buen estado general, con diagnóstico de enfermedad gingival inducida por placa que acude al Servicio de Periodoncia de la Cínica dental de la Universidad Peruana Cayetano Heredia. En el examen clínico se observó encía marginal y papilar eritematosa y edematosa generalizada, con acúmulo de placa y sangrado al sondaje generalizado; el índice de placa según O’Leary fue de $98 \%$ y el índice de sangrado según Ainamo de 98\% (Figuras 1, 2). Al examen radiográfico se observa presencia de cortical ósea intacta (Fig. 3).

\section{Tratamiento}

En la fase inicial se incluyó motivación e instrucción de higiene oral con sus respectivos controles de placa bacteriana, seguido de profilaxis con alisado en ambas arcadas, lo cual luego de una semana y a la reevaluación se observó me-

Tabla 1. Características de la gingivitis asociada a la pubertad (1).

\begin{tabular}{|c|}
\hline 1. Placa presente en el margen gingival. \\
\hline 2. Respuesta inflamatoria pronunciada de la gingiva. \\
\hline $\begin{array}{l}\text { 3. Debe ser circumpuberal así como la designada por el estadio } 2 \text { de } \\
\text { Tanner o mayor: (niñas, estradiol } \geq 26 \mathrm{pmol} / \mathrm{L} \text {; niños, testosterona } \\
\geq 8,7 \mathrm{nmol} / \mathrm{L} \text { ). }\end{array}$ \\
\hline 4. Cambios del color de la encía. \\
\hline $\begin{array}{l}\text { 5. Cambios en el contorno gingival con posible modificación del } \\
\text { tamaño gingival. }\end{array}$ \\
\hline 6. Incremento en el exudado gingival. \\
\hline 7. Sangrado al estímulo. \\
\hline 8. Ausencia de pérdida de inserción. \\
\hline 9. Ausencia de pérdida ósea. \\
\hline 10. Reversible después de la pubertad. \\
\hline
\end{tabular}

Tabla 1: Ann Periodontol 1999;4:7-17.

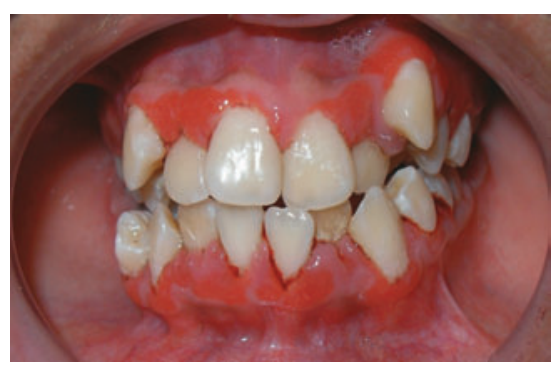

Fig 1. Fotografía frontal intraoral.

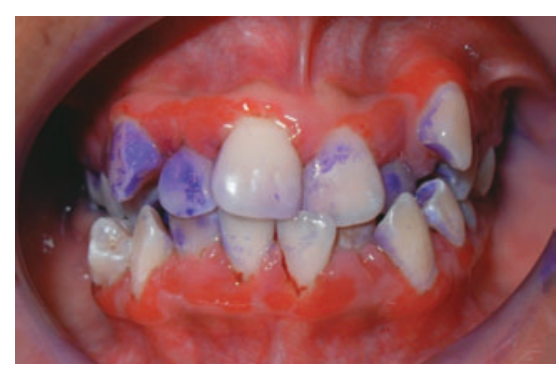

Fig 2. Indice de placa según O’Leary: 98\% e Indice de sangrado según Ainamo: 98\%

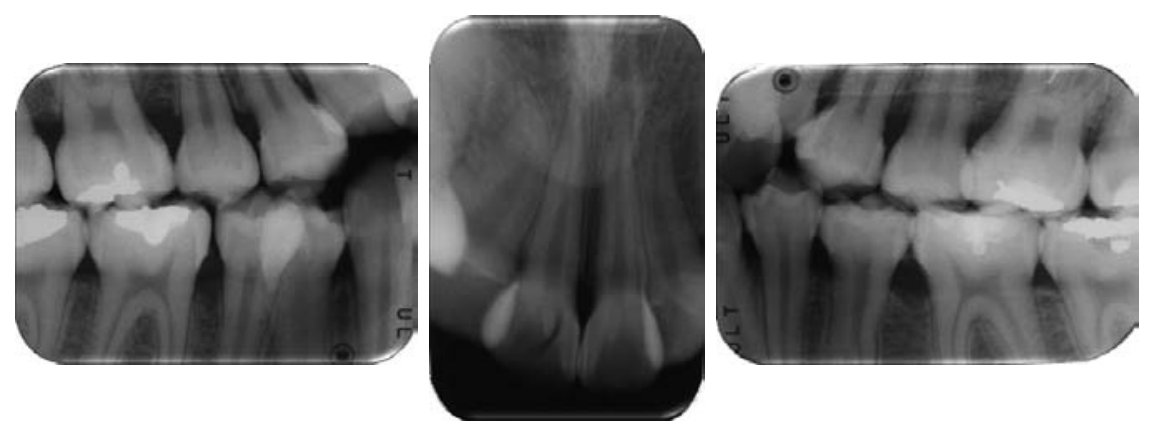

Fig 3 Radiografias periapical y bitewings de arcada superior e inferior.
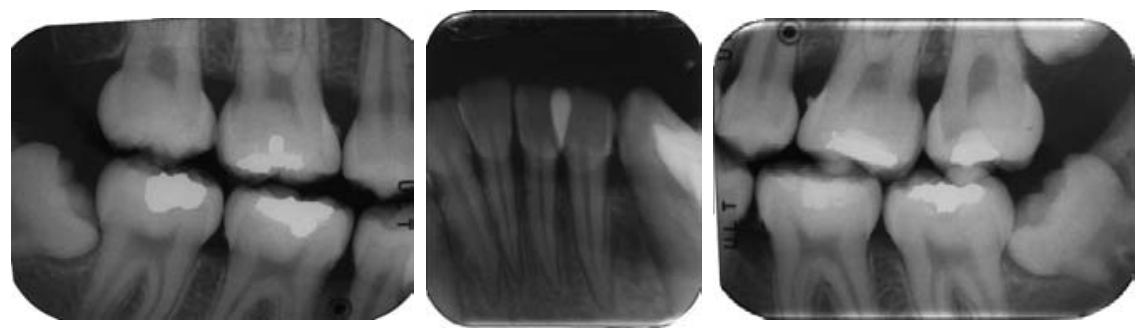

jorías tanto en los índices de placa como de sangrado y por ende una evolución favorable en cuanto a los parámetros clínicos periodontales.

La paciente presenta factores que predisponen a la enfermedad gingival, los cuales incluyen malposiciones dentarias y factores hormonales correspondientes a la edad; en cuanto a las malposiciones dentarias, la paciente será atendida en el servicio de ortodoncia para 


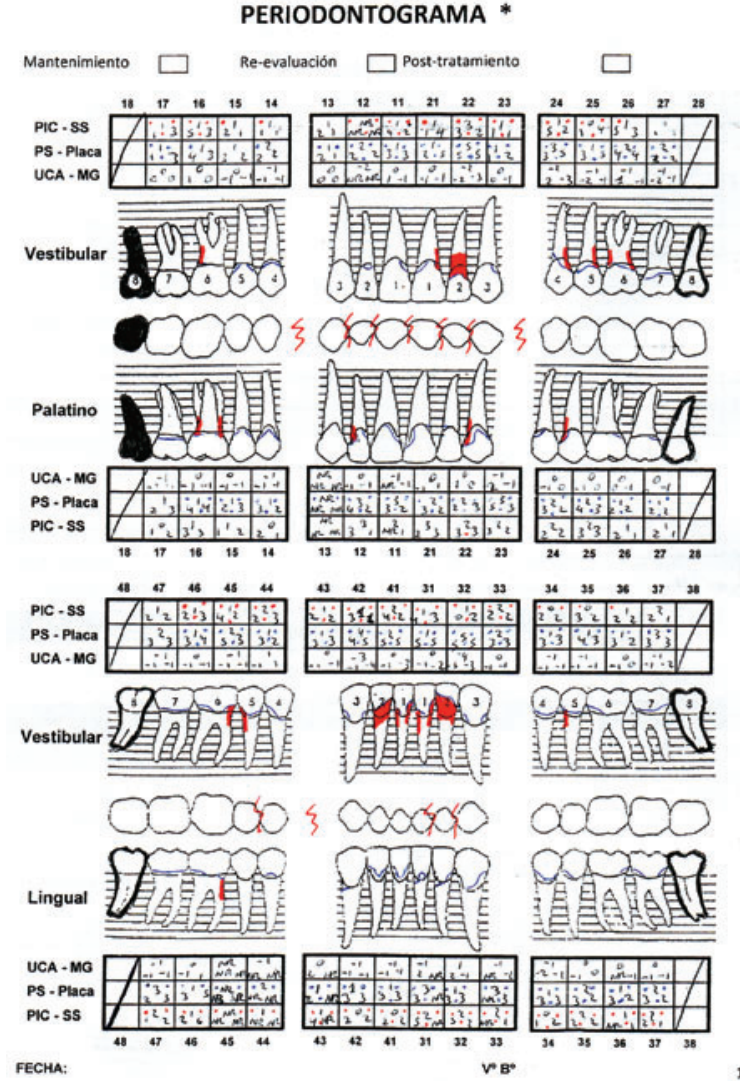

Fig 4. Periodontograma.

establecer una adecuada relacion interoclusal.

En cuanto a la fase quirúrgica se consideró la realización de una gingivoplastía a nivel de las piezas 32 y 42 con la finalidad de mejorar el contorno del margen gingival y facilitar la higiene a ese nivel (Fig. 4 y 5).

\section{Conclusiones.}

Las enfermedades gingivales son comunes en niños y adolescentes, por lo que se debe realizar una historia clínica completa y detallada para evaluar los factores que predisponen o modifican la enfermedad gingival tales como la edad, factores sistémicos, higiene oral, entre otros; para poder realizar un correcto diagnóstico y tomar en cuenta los diagnósticos diferenciales que podrían alterar nuestro plan de tratamiento. ${ }^{6}$

Los signos clínicos de la gingivitis asociada a la pubertad tiene mu-

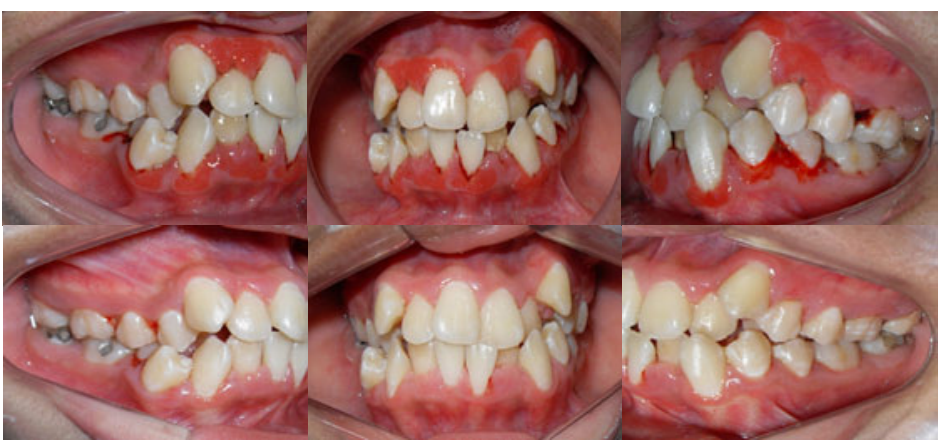

Fig 5. Fotos superiores iniciales y vistas inferiores correspondientes a la reevaluación; en las cuales se observa la mejoría evidente de los tejidos.

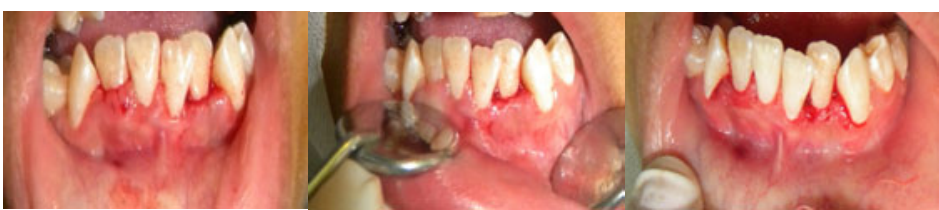

Fig 6. Fotos de la cirugía de gingivoplastía a nivel de piezas 32 y 42.

génesis de la enfermedad. ${ }^{8,9}$

La fluctuación normal y anormal de los niveles de hormonas incluye cambios en los niveles de hormona gonadotrópica durante la pubertad que pueden modificar la respuesta de inflamación gingival a la placa dental. ${ }^{10}$ Sin embargo, las condiciones gingivales usualmente responden a la remoción de los depósitos bacterianos y mejorar la higiene oral diaria. ${ }^{11,12,13}$

Usualmente, la inflamación periodontal durante la infancia está limitada a la encía y no resulta en pérdida de inserción o reabsorción de hueso alveolar. Sin embargo, la periodontitis agresiva causa pérdida de inserción y pérdida ósea en edad temprana, incluso en dientes deciduos. La fisiopatología de esta periodontitis no está bien entendida y por ende tampoco su tratamiento. Muchos estudios reportaron que el Aggregatibacter actinomycetecomitans (Aa) juega un rol importante en la periodontitis agresiva; sin embargo en algunos pacientes no se detecta el Aa, pero al análisis de la PCR revela al paciente positivo para Aa. ${ }^{14}$

La técnica de PCR es usada para 
detectar bacterias patógenas y es más sensitiva que los métodos de cultivo. ${ }^{14}$ Otros estudios sugirieron que el $\mathrm{C}$. gingivalis podría ser un patógeno de importancia para la periodontitis por su tripsina como proteasa. ${ }^{14}$

La alta prevalencia de la periodontitis agresiva estuvo también relacionada con la historia familiar. La frecuencia alta de la enfermedad en otros miembros de la familia sugiere una predisposición a la periodontitis agresiva. ${ }^{15}$

\section{Referencias Bibliograficas.}

1 Mariotti A. Dental Plaque - Induced Gingival Diseases. Ann Periodontol 1999; 4:7-19.

2 Theytaz GA, Christou P, Kiliaridis $\mathrm{S}$. Gingival changes and secondary tooth eruption in adolescents and adults: a longitudinal retrospective study. Am J Orthod Dentofacial Orthop. 2011, 139:S129-32.

3. Theytaz GA, Kiliaridis S. Gingival and dentofacial changes in adolescents and adults 2 to 10 years after orthodontic treatment. J Clin Periodontol. 2008 Sep;35:825-830.

4. Tiainen L, Asikainen S, Saxén L. Puberty-associated gingivitis. Community Dent Oral Epidemiol. 1992 Apr;20:87-9.

5. GN Güncü, TF Tözüm, F. Ça- glayan. (2005) Effects of endogenous sex hormones on the periodontium - Review of literature. Australian Dental Journal 50:3, 138-45

6. Wolfe MD, Carlos JP. Periodontal disease in adolescents: Epidemiologic findings in Navajo Indians. Community Dent Oral Epidemiol 1987;15:33-40.

7. Durward CS, Wright FA. The dental health of Indo-Chinese and Australian-born adolescents. Austr Dent J. 1989;34:233-9.

8. Nakagawa S, Fujii H, Machida Y, Okuda K. A longitudinal study from prepuberty to puberty of gingivitis. Correlation between the occurrence of Prevotella intermedia and sex hormones. J Clin Periodontol 1994;21:658-65.

9. American Academy of Periodontology - Research, Science and Therapy Committee Periodontal Diseases of Children and Adolescents. 1992, 1995, 2004.

10. Suzuki J, Okada M, Wang Y, Nii N, Miura K, Kozai K.. Localized aggressive periodontitis in Primary dentition: A case report. J Periodontol. July 2003, Vol. 74, No. 7: 1060-6.

11. Watanabe H, Umeda M, Seki
T, Ishikawa I. Clinical and laboratory studies of severe periodontal disease in adolescent associated with hypophosphatasia. A case report. J. Periodontol 1993; 64: 174-80.

12. Page RC, Bowen T, Altman L, et al. Prepuberal periodontitis I. Definition of a clinical disease entity. J Periodontol 1983; 54:257-71.

13. Leys EJ, Griffen AL, Strong SJ, Fuesrt PA. Detection and starin identification of Actinobacillus actinomycetemcomitans by nested PCR. J Clin. Microbiol 1994, 32: 1288-94.

14. Gazi MI, Cox SW, Clark DT, Eley BM. Characterization of protease activities in Capnocytophaga spp., Porphyromonas gingivalis, Prevotella spp., Treponema denticola and Actinobacillus actinomycetemcomitans. Oral Microbiol Immunol 1997;12:240-8.

15. Liran Levin, Valery Baev, Ron Lev, Ayala Stabholz and Malka Ashkenazi. Aggressive periodontitis among young Israeli army personnel. J. Periodontol 2006; 77:1392-6. 